\title{
Shoulder pain in a patient on haemodialysis
}

\author{
T R Goodman, E McNally
}

Nuffield Orthopaedic Centre NHS Trust, Oxford,

United Kingdom

T R Goodman

E McNally

^Department of

Diagnostic Imaging,

The Robert Jones and

Agnes Hunt Orthopaedic

Hospital,

Oswestry,

Shropshire SY10 7AG,

United Kingdom.

Correspondence to:

Dr T R Goodman,

Nuffield Orthopaedic Centre

NHS Trust,

Windmill Road,

Headington,

Oxford OX4 4LD

United Kingdom.

\section{Case history}

A forty eight year old man was referred with a three year history of right shoulder pain which was beginning to interfere with his job as a carpenter. The symptoms were not controlled by oral non-steroidal anti-inflammatory drugs.

Past history included renal failure secondary to chronic glomerulonephritis twenty years previously for which he had been receiving haemodialysis ever since.

Ten years previously he had a total parathyroidectomy due to tertiary hyperparathyroidism. Three years previously nerve conduction studies confirmed median nerve compression on the right for which he underwent carpal tunnel release.

Examination revealed soft tissue swelling around the shoulder together with a limited range of movement. A rotator cuff tear was considered and a plain radiograph (fig 1) and

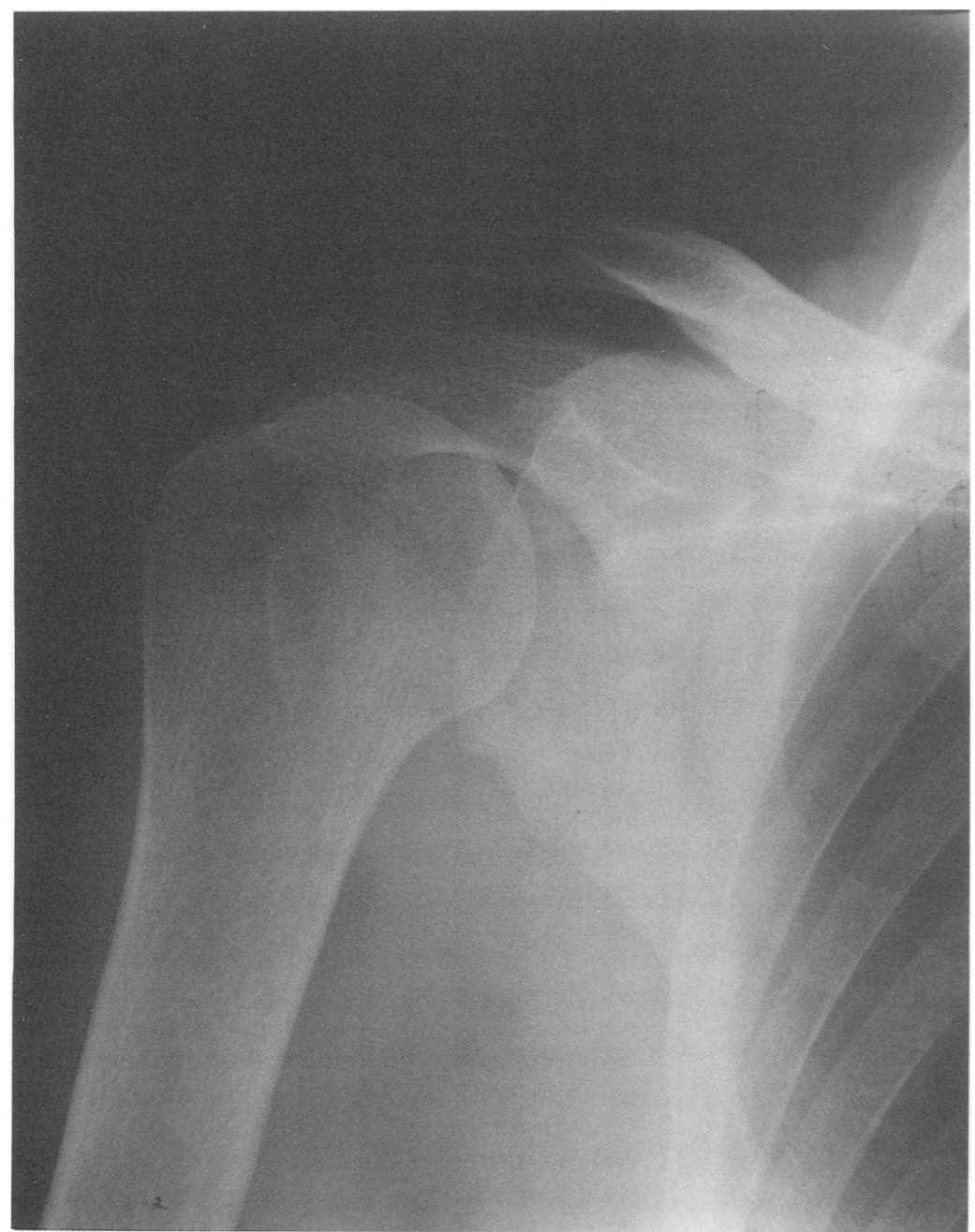

arthrogram (fig 2) of the shoulder were obtained.

\section{Questions}

1) What does the radiograph of the shoulder show?

2) What does the arthrogram of the shoulder show?

3) What diagnoses would you consider?

4) What other imaging might help to distinguish between these?

\section{Answers}

1) The anterio-posterior view of the right shoulder shows a reduction in the distance between the acromion and the humeral head with associated subacromial humeral sclerotic change. These findings are non-specific but suggest rotator cuff impingement by the acromion process. This impingement of the acromion through the rotator cuff onto the humeral head causes muscle thinning, tearing and humeral head insult giving rise to the reduction in acromiohumeral distance and humeral head sclerosis respectively. Other associated plain radiograph findings in rotator cuff tears are sclerosis and loss of the normal convex shape of the inferior surface of the acromion. However, these signs are also non specific.

2) There is no rotator cuff tear. No contrast is seen passing into the subacromial bursa nor the subdeltoid bursa making a complete rotator cuff tear impossible. There are, however, multiple villous filling defects within the joint. These filling defects are an unusual appearance but are likely to be synovial in origin.

\section{3) Amyloid deposition}

Pigmented villonodular synovitis

Rheumatoid arthritis

Synovial chondromatosis

In view of the chronic haemodialysis history, dialysis related amyloid arthropathy is the most likely diagnosis. Pigmented villonodular synovitis is a less likely cause as it is a rare disorder which affects younger patients and most commonly involves the knee. In rheumatoid arthritis the diagnosis would have been made on clinical examination and plain film changes would be expected after such a long history. Synovial thickening does occur 


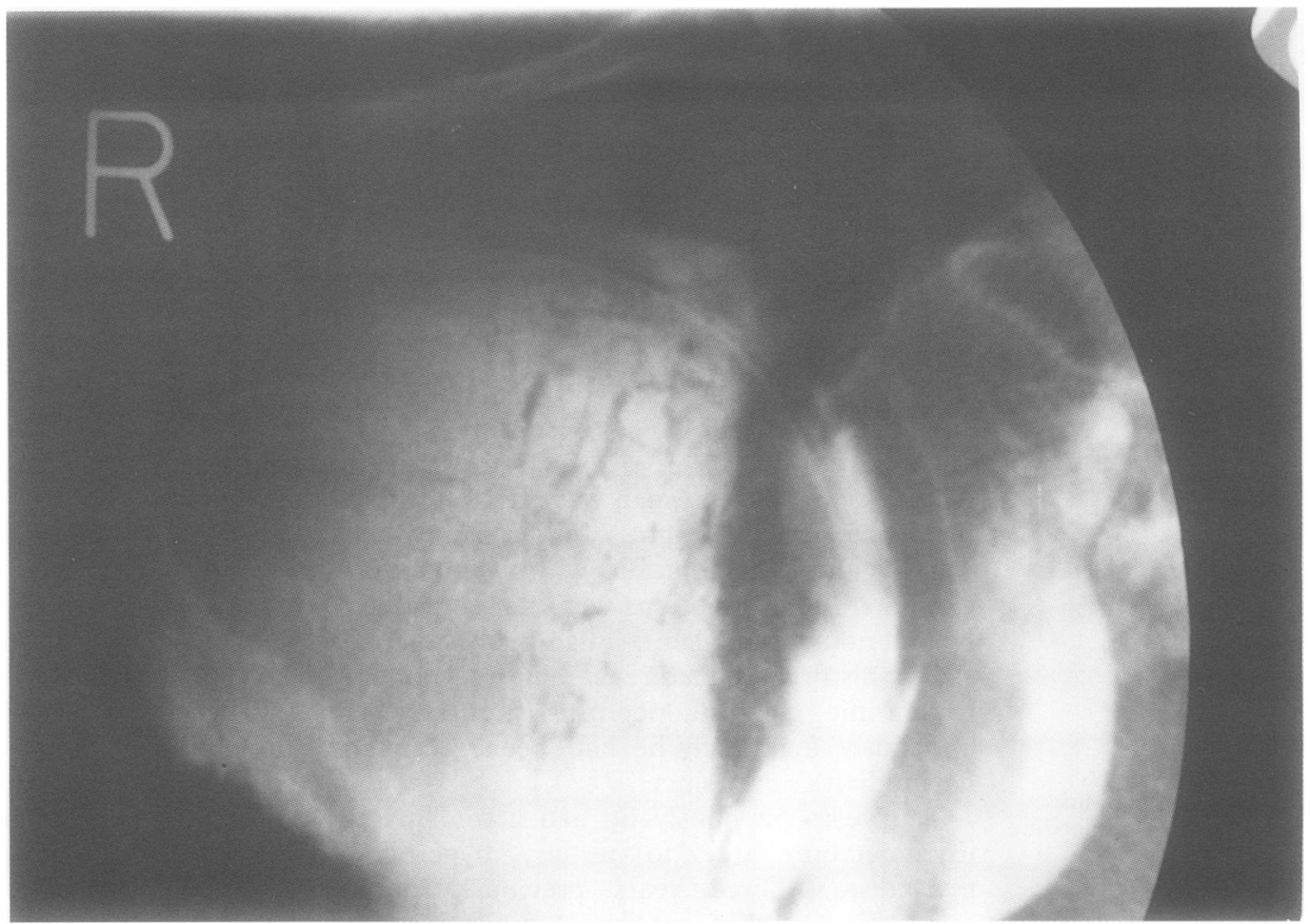

Figure 2 Arthrogram of the right shoulder.

cartilaginous loose bodies might be expected on arthrography. Hyperparathyroidism, which is a likely diagnosis in a patient on chronic haemodialysis would not give this appearance. Hyperparathyroidism classically produces subperiosteal, subchondral and subligamentous bone resorption none of which are seen in this case. Calcium pyrophosphate deposition disease is associated with hyperparathyroidism and commonly affects the shoulder but one would expect to see chondrocalcinosis on the plain radiograph.

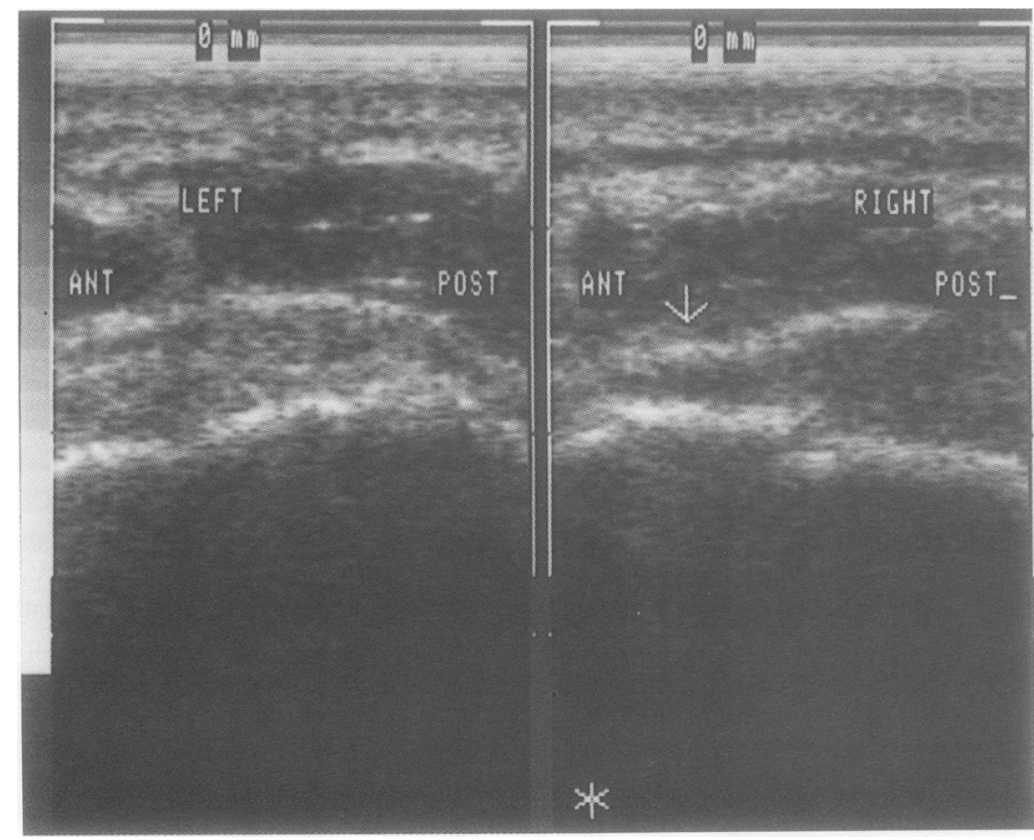

Figure 3 Ultrasound scan of shoulders (not this case) with a normal rotator cuff demonstrated on the left. On the right there is a thinning of the cuff around a hypoechoic region indicating a tear (arrow).
4) ULTRASONOGRAPHY

Magnetic resonance imaging (MRI)

Bone scintigraphy and computed tomography, although useful in imaging boney structures play no role in the assessment of the rotator cuff or internal apparatus of the joint itself.

Ultrasonography is being increasingly used as a fast, cheap and non-invasive technique for imaging shoulders and the rotator cuff in particular. Using the asymptomatic shoulder as a reference standard and the shoulder held in internal rotation, a linear array probe can image most of the rotator cuff, a small proportion being impossible to see under the acromion process. Tears can be identified as hypoechoic discontinuity in the cuff (fig 3 ), a hyperechoic focus within the cuff representing inflammatory tissue or a non-visualised cuff. Various centres have published encouraging results with this technique, with a correlation with arthrography in the detection of tears of up to $93 \% .^{8}$

The most useful investigation, however, would be an MRI scan. Using a Siemans 1.5 Tesla unit, T1 and T2 weighted oblique coronal images were obtained (figs 4,5 and 6).

These images confirm an intact supraspinatus tendon (thick arrows fig 4 ). There is an area of high signal beneath the acromion on the T2 weighted image (thick arrow fig 6) which represents fluid in the subacromial bursa. The most striking feature is the widening of the glenohumeral joint space due to marked synovial proliferation. This synovium is of intermediate to low signal on $\mathrm{T} 1$ weighted images and of low signal on T2 weighted images (thin arrows figs 4 and 6). There is no associated effusion but the humeral head contains at least two erosions of similar 


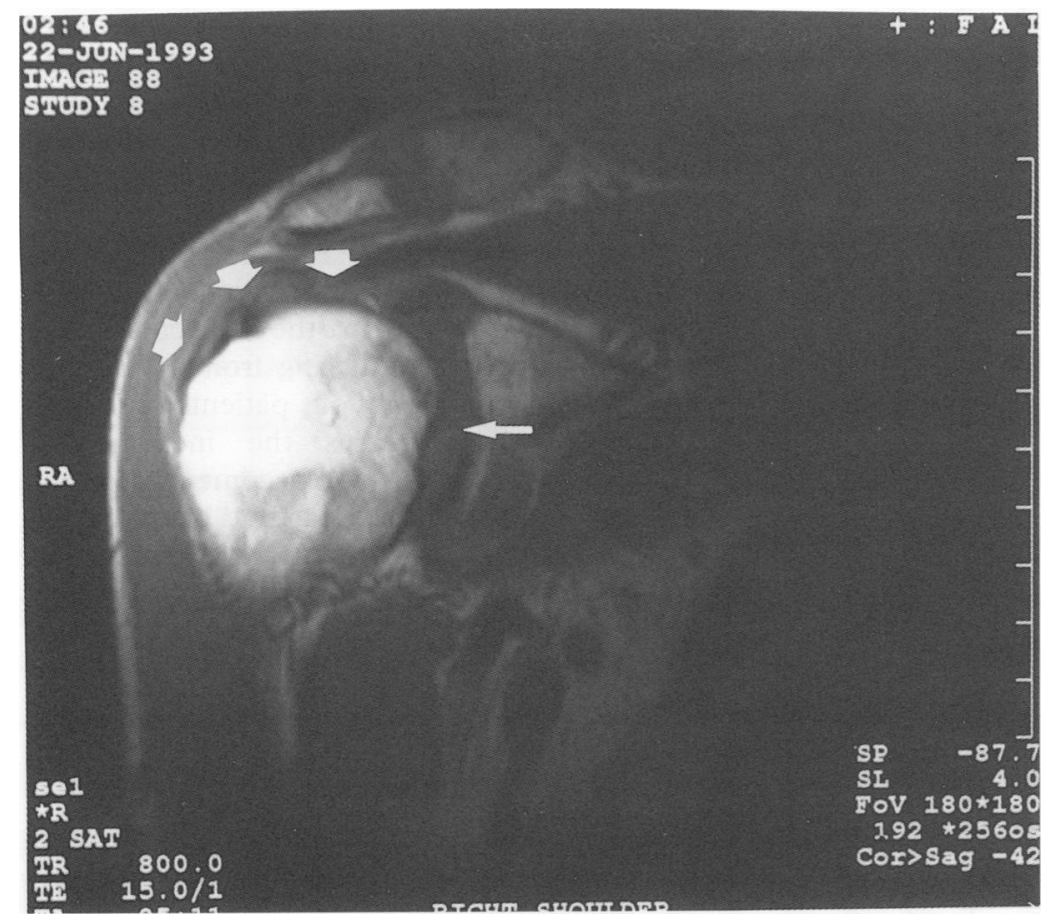

Figure $4 T 1$ weighted oblique coronal MRI section of the right shoulder. Thick arrows show intact supraspinatus tendon. This arrow demonstrates widened glenohumeral joint space containing material of low/intermediate signal.

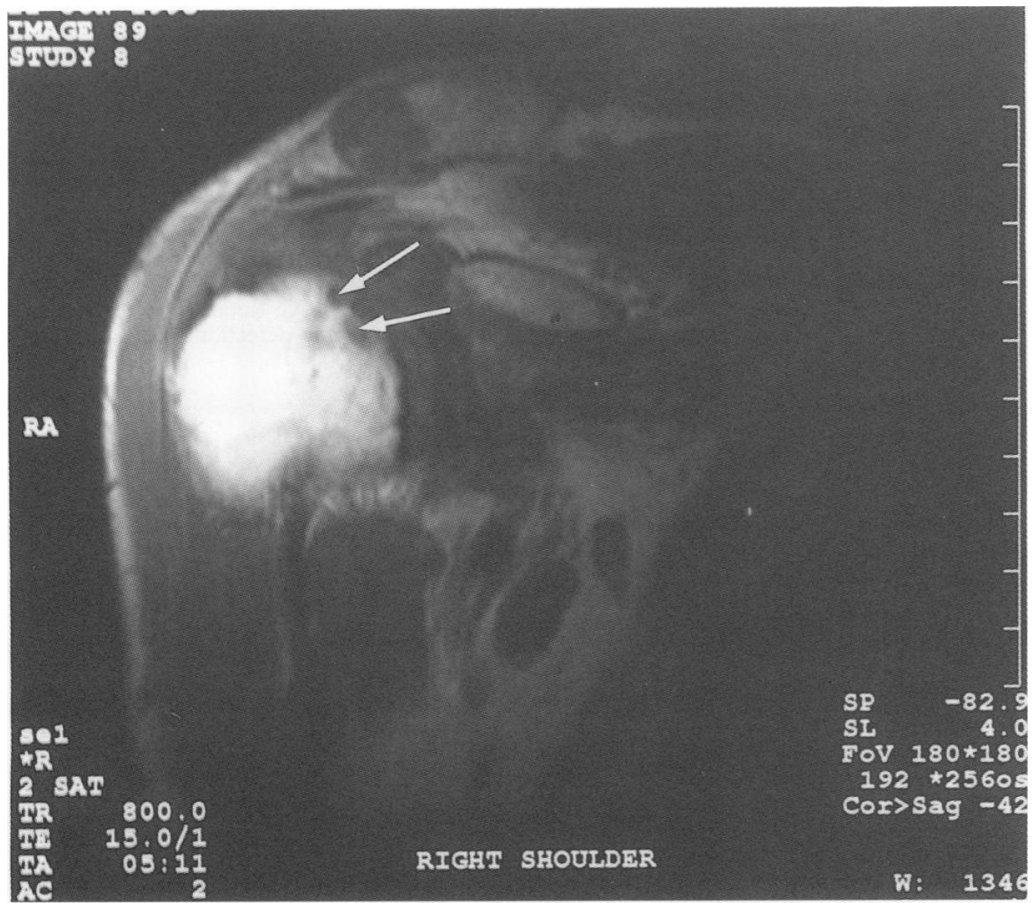

Figure $5 T 1$ weighted oblique coronal MRI section of the right shoulder. Arrows show humeral head erosions containing material of similar signal characteristics to that in the joint space. only recently been elucidated. In 1987 a characteristic B2-microglobulin amyloid tissue was shown to be the cause ${ }^{1}$ and the condition was renamed haemodialysis related amyloid arthropathy (HRAA). Descriptions of its radiological and magnetic resonance appearances are few, despite the fact that it is a common disorder.

The B2-microglobulin protein fibril does not pass through cellulose dialysis membranes. Plasma levels in patients receiving haemodialysis can therefore be between eight and 60 times higher than controls. ${ }^{1}$ This excess plasma protein can precipitate out and form the characteristic B2-microglobulin amyloid tissue. Unlike primary or secondary amyloid where joint involvement is relatively rare, haemodialysis related amyloidosis appears to have a predilection for the musculoskeletal system. Deposits can be laid down in bone, tendons, ligaments, synovium, cartilage, intervertebral discs and surrounding soft tissues. ${ }^{2}$ Because of this widespread amyloid deposition presentation can be varied.

The condition is characterised by pain and stiffness, usually of the shoulders, but also affecting the wrists, knees, hips and other large joints. ${ }^{3}$ Later findings include trigger fingers, tendon contracture or rupture and pathological fractures through bone cysts, usually in the femoral neck. ${ }^{4}$ Symptoms are rare in the first five years after haemodialysis starts and carpal tunnel syndrome usually precedes its development. $^{5}$

There are no plain radiographic changes in HRAA until late in the disease process. A destructive arthropathy eventually develops with juxta-articular cystic radiolucencies of the affected bones. This is commonly associated with surrounding soft tissue masses. A characteristic feature is the preservation until late in the disease of the affected joint space.

Bone cysts occur in $51 \%$ of patients with HRAA $^{4}$ and if in the femoral neck usually account for the pathological fractures seen in this condition. However, most cysts are asymptomatic and are incidental findings in the carpals and phalanges.

MRI has played a useful role in studying and understanding HRAA. Amyloid has a characteristic low signal on T2 weighted sequences and of intermediate to low signal on $\mathrm{T} 1$ weighted sequences due to its long $\mathrm{T} 1$ and short $\mathrm{T} 2$ relaxation times. ${ }^{5-7}$ This is most likely due to the protons being held relatively fixed within the characteristic beta-pleated sheet resulting in quick phase dispersion of the magnetic signal. This low T2 signal helps distinguish amyloid from acute synovitis, inflammatory masses or Brown's tumour of hyperparathyroidism. Haemosiderin has similar signal characteristics to amyloid. Consequently, conditions where haemosiderin may be contained in the joint, such as a bleeding diathesis associated haemarthrosis, or pigmented villonodular synovitis (PVNS) would give similar appearances. In haemophilia the joint space is reduced and new bone formation occurs, unlike HRAA. A biopsy may be required to distinguish PVNS from HRAA.
Haemodialysis related arthropathy has long been recognised but its pathoaetiology has 


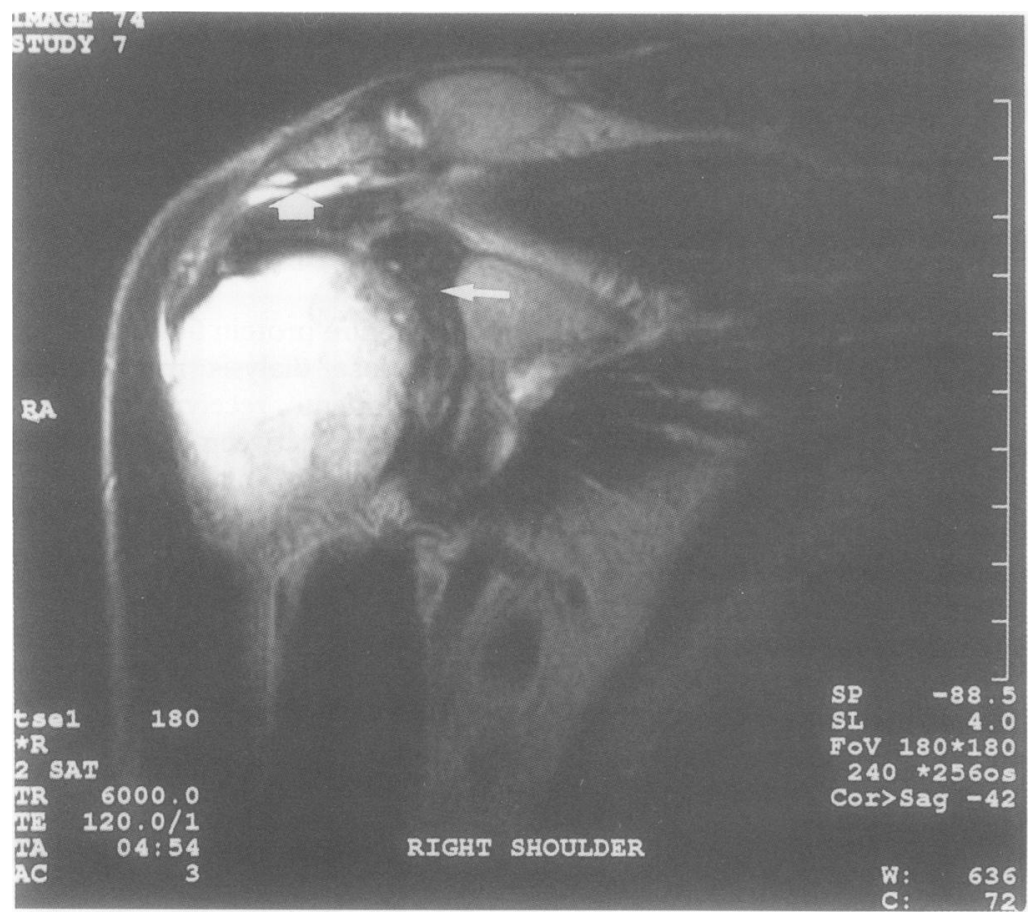

Figure 6 T2 weighted oblique coronal MRI section of the right shoulder. Thin arrow shows widened glenohumeral joint space with material of low signal. Thick arrow shows high signal beneath the acromion representing fuid in the subacromial bursa.

The MRI appearances of synovial chondromatosis are distinctive. Multiple well defined fragments of low to intermediate signal intensity of synovial origin are seen in the joint which usually contains an effusion.

Of the few reports that have been published none demonstrate MRI contrast agents to be of use in the imaging of amyloid tissue.

The relatively few affected large joints that have been reported show massive amyloid involvement of the synovium and subarticular erosions, ${ }^{5}$ as in this case. It may be that the preservation of joint space until late in the disease is a reflection of the space occupying effect of this bulky synovium. No studies have been performed to date to confirm this.

MRI undoubtedly has much to offer in determining the extent and involvement of amyloid deposition in the musculoskeletal system of patients suffering from HRAA. As the life expectancy of patients receiving haemodialysis increases the incidence of HRAA will rise. MRI will become increasingly helpful as a noninvasive method of diagnosing the condition and as further developments are made in the treatment of HRAA, magnetic resonance could provide a useful means of monitoring responses.

1 Bardin B, Zingraff J, Shirahama T, et al. Haemodialysisassociated amyloidosis and beta-2 microglobulin. $A m \mathcal{F}$ Med 1987; 83: 419-24.

2 Kessler M, Netter P, Axoulay E, et al. Dialysis-associated arthropathy: a multicentre survey of 171 patients receiving haemodialysis for over 10 years. $\mathrm{Br} \mathcal{F}$ Rheumatol 1992; 31: 157-62.

3 Sargent M A, Fleming S J, Chattopadhyay C, et al. Bone cysts and haemodialysis-related amyloidosis. Clin Radiol 1989; 40: 277-81.

4 Kurer M H J, Baillod R A, Madgwick J C A. Musculoskeletal manifestations of amyloidosis. $f$ Bone foint Surg 1991; 73-B: 271-6.

5 Cobby $M$ J, Adler R S, Swartz R, et al. Dialysis-related amyloid arthropathy: MR findings in four patients. $A m \mathcal{F}$ Roentgenology 1991; 157: 1023-7.

6 Tagliabue J R, Stull M A, Lack E E, et al. Case report: amyloid arthropathy of the left ankle. Skeletal Radiol 1990; 19: 448-52.

7 Olliff J F C, Hardy J R, Williams M P, et al. Case report: magnetic resonance imaging of spinal amyloid. Clin Radiol 1989; 40: 632-3.

8 Soble M G, Kaye A D, Guay R C. Rotator cuff tear: clinical experience with sonographic detection. Radiology 1989; 173: $319-21$. 